\title{
The locus of perceived equidistance in binocular vision
}

\author{
JÜRGEN HELLER \\ Justus-Liebig-Universität Giessen, Giessen, Germany
}

\begin{abstract}
Empirical studies of the locus of perceived equidistance in binocular vision have revealed a characteristic change of its form, depending on absolute distance. This result is commonly taken to indicate influence of vergence-related binocular information, a conclusion that is by no means exclusively dictated by the data. Heller (1997) has suggested an alternative theoretical account that is based on the idea of independently combining the outcome of monocular input transformations without any form of binocular interaction. This article provides an experimental test of the structural assumption lying at the core of the axiomatic foundation of Heller's theory. I test the so-called Reidemeister condition under reduced cue conditions in two settings for each of 7 subjects. The results provide strong evidence for the validity of the Reidemeister condition and thus challenge the view that the locus of perceived equidistance depends on vergence-related binocular information. The discussion of the factors contributing to the monocular input transformations emphasizes the role of the optical properties of the eyes.
\end{abstract}

Egocentric distance is assumed to play a central role in visual spatial orientation, especially under reduced cue conditions. The present article will focus on the locus of perceived equidistance (LPED) in binocular vision, which is constituted by the points in space that are apparently located at a constant radial distance from the observer. Various empirical studies have revealed a characteristic change of the form of the LPED with increasing absolute distance (e.g., Foley, 1966, 1970; Hardy, Rand, Rittler, Blank, \& Boeder, 1953), which has been attributed to the influence of vergence-related binocular information (Foley, 1978, 1980). It will be shown that this interpretation is by no means exclusively dictated by the data. An alternative explanation considers the LPED as arising from an independent combination of monocular information. The precise nature of this independent combination is captured by a formal theory (Heller, 1997), which has been shown to follow logically from a set of qualitative axioms. The purpose of the present study is to perform an experimental test of the structural assumption lying at the core of this axiomatic approach.

\section{Preliminaries and Previous Work}

Theories of binocular space perception seek to predict perceived spatial relations, given the coordinates of the respective stimuli in physical space. Nearly all of them

I am grateful to Cornelia Retzer for her assistance in conducting the experiment. Correspondence concerning this article should be addressed to J. Heller, Institut für Psychologie, Karl-Franzens-Universität Graz, Universitätsplatz 2, A-8010 Graz, Austria (e-mail: juergen.heller@, uni-graz.at).

Note-This article was accepted by the previous editorial team, headed by Neil Macmillan. employ a bipolar coordinate frame, reflecting the fact that our eyes are looking at the world from two different vantage points. Usually, the position of the stimuli is specified using head-centric bipolar coordinates, with the rotation centers of the two eyes serving as the reference points. Figure 1A introduces two sets of bipolar coordinates in the horizontal plane at eye level, to which I will confine my consideration. The angles of monocular azimuth $\alpha$ and $\beta$ form a pair of monocular bipolar coordinates that characterize the lateral deviation from straight ahead (positive to the left, negative to the right) with respect to the right eye and the left eye. Most theories of binocular vision, however, refer to the binocular bipolar coordinates $\gamma$ and $\varphi$, which are related to $\alpha$ and $\beta$ by

$$
\gamma=\alpha-\beta \text { and } \varphi=\frac{\alpha+\beta}{2} .
$$

The trajectories of constant binocular parallax $\gamma$ are known as the Vieth-Müller circles, while the trajectories of constant binocular azimuth $\varphi$ are called the hyperbolas of Hillebrand (see Figure 1B). Notice that the binocular parallax $\gamma$ characterizing a Vieth-Müller circle may be interpreted as the vergence angle that the visual axes subtend when any point on it is fixated.

The monocular bipolar coordinates $\alpha$ and $\beta$ are commonly considered direct indication of the locus of the proximal stimulus - namely, the retinal image. This interpretation, however, heavily draws upon an idealization of the relevant optics by identifying the center of rotation and the optical node with the center of curvature of a spherical eye. On the basis of this simplification, the Vieth-Müller circle through the fixation point is considered the theoretical horizontal horopter; that is, the locus of stimuli that are projected onto corresponding retinal points (points that are congruent when the two retinas are superimposed). In 

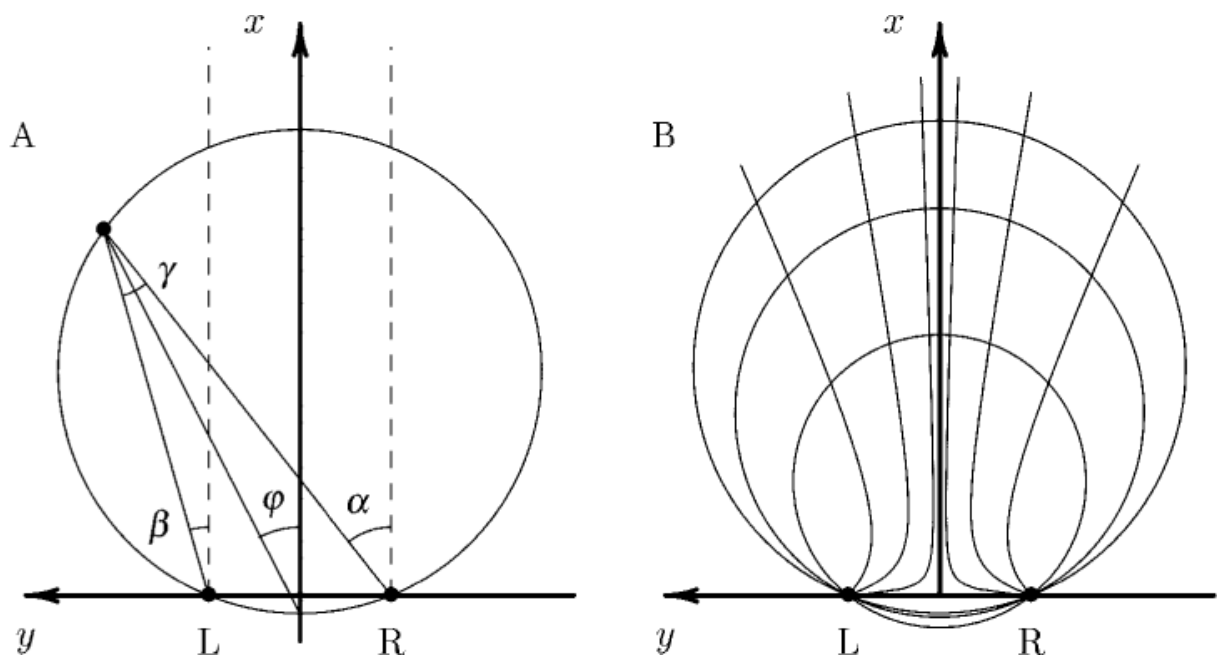

Figure 1. (A) The angular coordinates $\alpha$ and $\beta$ denote monocular directions with respect to the right eye $(R)$ and the left eye $(L)$, relative to the dashed lines parallel to the $x$ axis (positive to the left, negative to the right). The bipolar parallax $\gamma$ associated to a stimulus is the angle subtended by the visual axes when the eyes converge on the stimulus. The bipolar latitude $\varphi$ describes the lateral deviation of a stimulus from the $x$ axis. (B) Vieth-Müller circles $\gamma=\alpha-\beta=$ constant and hyperbolas of Hillebrand $\varphi=(\alpha+\beta) / 2=$ constant for different values of the respective constants.

other words, the parallax difference exhibited by two points can be identified with the horizontal disparity induced by them. Accordingly, the hyperbola of Hillebrand is the locus of symmetric retinal points, which deviate to the same extent but in opposite directions from the foveas.

The idea that the LPED coincides with the Vieth-Müller circle is fundamental to the seminal work of Luneburg (1947) in which he related depth perception to a nonEuclidean geometrical structure of binocular visual space. The assumption that any two points on the Vieth-Müller circle are perceived at the same egocentric distance is motivated by the fact that, if idealizing the optics of the eye as described above, these points induce zero retinal disparity. The Luneburg theory had an enormous impact on the research in the field, and a large number of experimental investigations have been devoted to an empirical test of its basic assumptions and consequences (see Indow, 1991, for a review). Their results, besides revealing remarkable individual differences, provide ample evidence that the LPED exhibits systematic deviations from the Vieth-Müller circle. In experiments presenting real point sources of light, the LPED shows a tendency toward physical equidistance and may be considerably skewed with respect to the median plane (Foley, 1966; Hardy et al., 1953; see present Figure 4). Discrepancies also result with stereoscopic presentation. However, in this case the LPED turns out to be more concave than the ViethMüller circle (Foley, 1970). Despite the different shapes of the LPED in these studies, the resulting parallax differences to the Vieth-Müller circle do not remain constant but tend to decrease in size with increasing distance. This means that the LPED is characterized by a pattern of parallax differences that varies with the ab- solute binocular parallax of the reference point. Identifying binocular parallax $\gamma$ with the vergence angle that the visual axes subtend when the respective point is fixated led to the conclusion that vergence is involved in judging equidistance (Foley, 1978, 1980). It is assumed that the parallax differences (to the Vieth-Müller circle defined by the reference point), commonly identified with the horizontal retinal disparities, are evaluated on the basis of oculomotor cues related to vergence. This account of the LPED, however, seems to conflict with the widely accepted view that vergence is not a reliable depth cue, at least beyond a distance of about $2 \mathrm{~m}$ (e.g., Howard \& Rogers, 1995; Mon-Williams \& Tresilian, 1999). Below, I develop an alternative explanation of the LPED that works without referring to vergence-related binocular information, but exclusively rests upon an independent combination of monocular information.

\section{A Theoretical Account of the LPED}

Most formal treatments of the LPED, like those of Blank (1978) and Foley $(1978,1980)$, are cast within the binocular bipolar coordinate frame. The $\gamma$ and $\varphi$ coordinates seem to be the natural choice for the Luneburg theory (Luneburg, 1947), which assumes that perceived distance only depends on $\gamma$, and that perceived direction only depends on $\varphi$. The above mentioned experimental results concerning the LPED, however, cast doubt on the general usefulness of these coordinates. Moreover, the often observed skewedness of the LPED with respect to the median plane (e.g., Foley, 1966), which is most likely due to monocular effects (e.g., aniseikonia), cannot be accounted for in a straightforward way within the binocular bipolar coordinate frame. 
The subsequently suggested theory is formulated on the basis of the monocular bipolar coordinates, where each stimulus is characterized by a pair $(\alpha, \beta)$ of monocular azimuth angles (see Figure 1A). By $(\alpha, \beta) \sim\left(\alpha^{\prime}, \beta^{\prime}\right)$, I will denote that the stimuli $(\alpha, \beta)$ and $\left(\alpha^{\prime}, \beta^{\prime}\right)$ are judged to be equidistant. Luneburg's assumption that the LPED is the Vieth-Müller circle - that is, the trajectory $\gamma=\alpha-\beta=$ constant - then reads

$$
(\alpha, \beta) \sim\left(\alpha^{\prime}, \beta^{\prime}\right) \text { iff } \alpha-\beta=\alpha^{\prime}-\beta^{\prime} .
$$

Notice that Equation 2 identifies the perceptually relevant magnitudes with the monocular bipolar coordinates $\alpha, \beta$. It formally characterizes the LPED as the zero-disparity horopter-that is, the trajectory satisfying $\gamma-\gamma^{\prime}=(\alpha$ $-\beta)-\left(\alpha^{\prime}-\beta^{\prime}\right)=0$. A generalization of Luneburg's theory is obtained if the perceptually relevant magnitudes are conceived as functions of $\alpha$ and $\beta$ rather than being identified with them. Within such an approach, the observed discrepancy between the LPED and the ViethMüller circle is assumed to result from input transformations which operate on the monocular information. The effects captured by those transformations may be caused by optical properties of the eye, such as differing magnification factors of the lenses or by the retinas deviating from a spherical form. The transformations may also capture effects of the neural processing that occurs prior to binocular combination. These ideas are expressed more precisely by stating them in a formal way.

I assume that there exist (strictly increasing) functions $f$ and $g$, such that for all stimuli $(\alpha, \beta),\left(\alpha^{\prime}, \beta^{\prime}\right)$,

$$
(\alpha, \beta) \sim\left(\alpha^{\prime}, \beta^{\prime}\right) \text { iff } f(\alpha)-g(\beta)=f\left(\alpha^{\prime}\right)-g\left(\beta^{\prime}\right) .
$$

The monocular input transformations $f$ and $g$ in Equation 3 characterize the perceptually relevant magnitudes as functions of the position of the distal stimulus relative to the right eye and the left eye, respectively. The above mentioned interpretation of these transformations suggests an observer-specific choice of these functions, which is in line with the observed individual differences concerning the LPED.

The contributions of both eyes are assumed to combine in an independent way, without any form of binocular interaction. This becomes obvious if we rearrange the terms in Equation 3 to obtain

$$
(\alpha, \beta) \sim\left(\alpha^{\prime}, \beta^{\prime}\right) \text { iff } g(\beta)-g\left(\beta^{\prime}\right)=f(\alpha)-f\left(\alpha^{\prime}\right) .
$$

The left-hand side of the representing equation depends only on information coming from the left eye, while the right-hand side depends only on information coming from the right eye. Thus, according to Equations 3 and 4, judgments of equidistance result from simply comparing both monocular contributions and do not draw upon any binocular information. Moreover, Equation 4 implies that the perceptually relevant monocular information does not depend on the absolute position of the stimuli. For any pair of stimuli with fixed coordinates $\beta$ and $\beta^{\prime}$, for example, the perceptually relevant information in the left eye [represented by the difference $g(\beta)-g\left(\beta^{\prime}\right)$ ] stays the same, regardless of the respective $\alpha$ coordinates - that is, regardless of the absolute position of the stimuli. This is in clear contrast to Foley's $(1978,1980)$ idea that disparities are evaluated on the basis of oculomotor cues related to vergence. If the observer converges on the stimuli (as will usually be the case whenever the eyes are allowed to move freely), by changing their absolute position a reevaluation of the monocular information is predicted by Foley's theory, even if the $\beta$ coordinates remain constant.

In principle there are two different ways to check the validity of the theory formalized by Equation 3. One strategy requires the introduction of a priori assumptions as to the form of the functions $f$ and $g$ in order to derive a concrete prediction of the LPED that may be tested against data by a global goodness-of-fit test. The scope of such a test, however, is clearly limited by the empirical adequacy of these additional assumptions. I thus prefer to follow a different strategy that allows for testing the theory in its general form and tries to pin down the functions in a second step. Such a general test can be developed within a measurement-theoretic approach (cf. Krantz, Luce, Suppes, \& Tversky, 1971). Heller (1997) provided a set of qualitative axioms that are sufficient for proving the existence of functions $f$ and $g$, satisfying a slightly more general version of Equation 3. The key property in this set of axioms is the so-called Reidemeister condition, a property that is well known in the context of conjoint measurement (Krantz et al., 1971; Chapter 6). Given the judgments of equidistance

$$
\begin{aligned}
& \left(\alpha_{1}, \beta_{1}\right) \sim\left(\alpha_{0}, \beta_{0}\right) \\
& \left(\alpha_{1}, \beta_{3}\right) \sim\left(\alpha_{0}, \beta_{2}\right) \\
& \left(\alpha_{3}, \beta_{3}\right) \sim\left(\alpha_{2}, \beta_{2}\right),
\end{aligned}
$$

the Reidemeister condition requires that

$$
\left(\alpha_{3}, \beta_{1}\right) \sim\left(\alpha_{2}, \beta_{0}\right)
$$

holds. It is easily seen that the Reidemeister condition is a necessary consequence of the proposed theory. By applying Equation 4 to the indifferences R1, R2, and R3, we obtain the equation at the bottom of the page. This sequence of equations implies equality of the leftmost and rightmost expressions, which is equivalent to R4.

The Reidemeister condition can be interpreted as a direct test of the assumption that the perceptually relevant monocular information (induced by a pair of $\alpha$ coordinates in the right eye and a pair of $\beta$ coordinates in the left eye) does not depend on the absolute position of the stimuli. The indifferences R1 and R2 state that the monoc-

$$
g\left(\beta_{1}\right)-g\left(\beta_{0}\right) \stackrel{(\mathrm{R} 1)}{=} f\left(\alpha_{1}\right)-f\left(\alpha_{0}\right) \stackrel{(\mathrm{R} 2)}{=} g\left(\beta_{3}\right)-g\left(\beta_{2}\right) \stackrel{(\mathrm{R} 3)}{=} f\left(\alpha_{3}\right)-f\left(\alpha_{2}\right)
$$


ular information induced by the pairs $\beta_{0}, \beta_{1}$ and $\beta_{2}, \beta_{3}$ both match the one induced by $\alpha_{0}, \alpha_{1}$. If now the pair $\alpha_{2}, \alpha_{3}$ also matches $\beta_{2}, \beta_{3}$, as requested in indifference $\mathrm{R} 3$, it also has to match the monocular information induced by $\beta_{0}, \beta_{1}$ (indifference R4). In its illustration in Figure 2, the Reidemeister condition translates into a nice geometrical property. The trajectories of the monocular bipolar coordinates involved in the indifferences $\mathrm{R} 1$ to R4 form two quadrangles (their corners are indicated by circles, and by triangles and squares, respectively, in Figure 2). If three pairs of stimuli, represented by corresponding corners of these two quadrangles, are each judged to be equidistant, so are the stimuli represented by the remaining pair of corners.

The subsequently described experiment was designed as an experimental test of the Reidemeister condition and implements the following rationale. If the coordinates $\alpha_{0}, \alpha_{1}, \alpha_{2}, \beta_{0}$, and $\beta_{2}$ are fixed in the above indifferences, the monocular azimuth $\alpha_{3}$ can be empirically determined in two independent ways. First, the coordi- nate $\beta_{1}$ is chosen to satisfy the indifference $\mathrm{R} 1$, and then the estimate $\alpha_{3}^{1}$ of $\alpha_{3}$ is determined according to indifference (R4). (Note that the superscript on $\alpha_{3}$ identifies whether it is the first or second estimate of $\alpha_{3}$.) Second, after getting $\beta_{3}$ from the indifference R2 an independent estimate $\alpha_{3}^{2}$ of $\alpha_{3}$ emerges from indifference R3. The Reidemeister condition then forces the two estimates to coincide - that is, it is equivalent to the equation $\Delta \alpha=$ $\alpha_{3}^{1}-\alpha_{3}^{2}=0$.

\section{METHOD}

\section{Subjects}

Seven female undergraduate students, 20-39 years of age, participated in the experiment. All subjects were naive with respect to the present study and had normal (Subjects $1-4,6,7$ ) or correctedto-normal (Subject 5) vision.

\section{Apparatus}

The computer-controlled experimental setup implemented the viewing conditions of primitive visual space (Roberts \& Suppes,

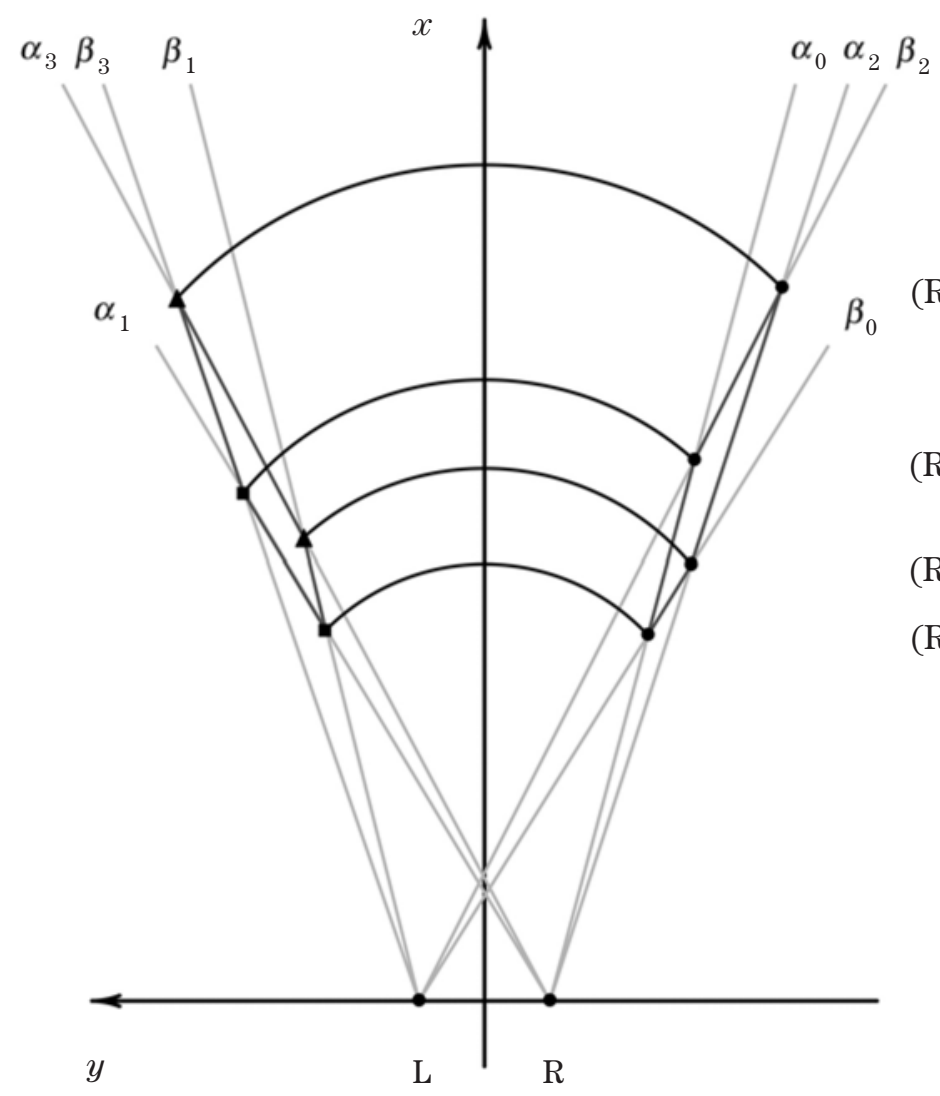

Figure 2. Graphical illustration of the Reidemeister condition. The points on the right-and thus the coordinates $\alpha_{0}, \alpha_{2}, \beta_{0}$, and $\beta_{2}$-are fixed in both settings. In setting $\Delta \alpha$, first the points represented by squares are moved along the line defined by $\alpha_{1}$ in order to obtain the matches R1 and R2, which provides the coordinates $\beta_{1}$ and $\beta_{3}$. Then the points represented by triangles are moved along the lines defined by these angles until the matches R4 and R3 are obtained. If the Reidemeister condition holds, the resulting positions have identical $\alpha$ coordinates. Setting $\Delta \beta$ proceeds in an analogous way. 
1967). The experiment was carried out in darkness in a totally lightproof room, and the subjects were prevented from observing the apparatus before they had finished their last session. Red light-emitting diodes (LEDs) with a diameter of about $2 \mathrm{~mm}$ were presented as stimuli. The setup consisted of four LEDs mounted on tripods that were placed at fixed positions, and four movable LEDs mounted on mechanical units. Driven by step motors, which were serially connected to a digital port of a PC, the LEDs of these units could be moved within a range of $94.5 \mathrm{~cm}$ in the direction of the $x$ axis and a range of $87.6 \mathrm{~cm}$ in direction of the $y$ axis, with a resolution of about $.25 \mathrm{~mm}$. All LEDs were set to a constant luminous intensity of $9 \cdot 10^{-6} \mathrm{~cd}$. The subjects were exclusively presented with static stimulus configurations - that is, the LEDs were only moved when all of them were turned off. The head was held fixed by a rest supporting the chin and forehead, which was individually adjusted at the beginning of each session so that all stimuli were lying in the horizontal plane at eye level. The eyes were allowed to move freely. A panel with two response keys was set in front of the subject.

\section{Procedure}

At the beginning of each session the subject stayed in complete darkness for $8 \mathrm{~min}$ to allow for dark adaptation. In the subsequent trials, the subject was presented with a reference point in the right half of the visual field, and a test point in the left half. Within a yes-no paradigm, the task was to decide whether the perceived radial distance to the test point was larger than that to the reference point. Judgments were communicated by pressing the corresponding response key. The position of the test stimulus was varied within an adaptive procedure, which is known as accelerated stochastic approximation (Kesten, 1958), along a trajectory of constant coordinate $\alpha$ or $\beta$, respectively. Starting with a subject-specific initial value of 0.9-1.5 arcmin, the stepsize was decreased after each occurrence of a response change. The procedure was stopped after six response changes corresponding to a minimal stepsize of $0.15-0.25$ arcmin.

With this experimental setup, the Reidemeister condition was tested in two settings, which will be called $\Delta \alpha$ and $\Delta \beta$. The stimulus configurations were chosen to meet two constraints: They had to fall within the range investigated in most of the previous empirical studies of the LPED (e.g., Foley, 1966), and they had to be as large as possible to allow for a strong test of the Reidemeister condition. In order to properly place the limited range of the movable LEDs according to the individual judgments, the exact coordinates of some of the presented points had to be varied slightly over subjects. For all subjects and in both settings, the point $\left(\alpha_{2}, \beta_{0}\right)$ had fixed Cartesian coordinates $x=257.80 \mathrm{~cm}$ and $y=-82.60 \mathrm{~cm}$. The $x$ coordinate of $\left(\alpha_{0}, \beta_{2}\right)$ varied over subjects between $281.50 \mathrm{~cm}$ and $303.86 \mathrm{~cm}$, and its $y$ coordinate between $-88.20 \mathrm{~cm}$ and $-94.65 \mathrm{~cm}$. Converting these Cartesian coordinates into monocular bipolar coordinates, based on the individual interpupillary distance, also fixes points $\left(\alpha_{0}, \beta_{0}\right)$ and $\left(\alpha_{2}, \beta_{2}\right)$. In addition, in setting $\Delta \alpha$ the value $\alpha_{1}=10^{\circ}$ was chosen, while in setting $\Delta \beta$ the coordinate $\beta_{3}$ was set to $\beta_{3}=8.95^{\circ}$ for all subjects, except for Subject 5, where it was set to $\beta_{3}=8.84^{\circ}$.

Each session consisted of a complete test of the Reidemeister condition in either setting $\Delta \alpha$ or setting $\Delta \beta$. Sessions were divided into two parts. The first part in setting $\Delta \alpha$ consisted of two interleaved adaptive procedures for estimating $\beta_{1}$ and $\beta_{3}$ via the indifferences R1 and R2, respectively. In Figure 2, this corresponds to assessing the required matches by moving the points represented by squares along the line defined by $\alpha_{1}$. The obtained values were then used in the second part to determine the estimates $\alpha_{3}^{1}$ and $\alpha_{3}^{2}$ of $\alpha_{3}$ via R4 and R3, again by running two interleaved adaptive procedures. In Figure 2 this means that $\alpha_{3}^{1}$ was determined by moving the point (represented by a triangle) along the line defined by $\beta_{1}$ to find the match in R4, and $\alpha_{3}^{2}$ was determined by moving the point (again represented by a triangle) along the line defined by $\beta_{3}$ to find the match in R3. The difference $\Delta \beta$ was estimated in a similar way.
After fixing the coordinates $\alpha_{0}, \alpha_{2}, \beta_{0}, \beta_{2}$, and $\beta_{3}$, estimates of $\alpha_{1}$ and $\alpha_{3}$ were obtained via R2 and R3, respectively. These coordinates then entered into the independent determination of two estimates for $\beta_{1}$ via R1 and R4.

To keep their motivation at a high level, the subjects received feedback on the precision of their performance after each session. The measure of precision used was the average of the standard deviations of all the adaptive procedures within the session, which did not provide any directional information.

After three training sessions, each subject took part in as many sessions as were necessary to collect five estimates in each of the settings. Whenever an adaptive procedure required a point that could not be presented with the current experimental setup, all the results of the particular session were discarded, and the session was repeated with an appropriately modified setup as long as a total of 20 sessions was not exceeded. Due to this limit, there are only four estimates in setting $\Delta \alpha$ for Subjects 3 and 7. Each session lasted about $30 \mathrm{~min}$.

\section{RESULTS}

Figure 3 shows the results of Subject 6 (setting $\Delta \alpha$ ) and Subject 1 (setting $\Delta \beta$ ) in Cartesian coordinates. These data plots of two contrary cases are easy to read because it was not necessary to readjust the reference points (indicated by the symbols at the right) in the course of the experiment. Each of the corresponding symbols at the left represents a single-session estimate of the point of perceived equidistance. In both settings, the symbols $\triangle, \square, \bigcirc$, and $\diamond$ denote the points involved in the indifferences R1 to R4, respectively. The differences of the $\alpha$ coordinates of the points represented by $\diamond$ and $\bigcirc$ enter into the statistical test in setting $\Delta \alpha$. In setting $\Delta \beta$, the test is based on the differences of the $\beta$ coordinates of the points represented by the symbols $\triangle$ and $\diamond$. In any case, Figure 3 also illustrates the layout of the experimental setup, which covered a range of about $250 \mathrm{~cm}$ along the $x$ axis, and about $210 \mathrm{~cm}$ along the $y$ axis.

The difference $\Delta \alpha$ was computed for points that were nearly $200 \mathrm{~cm}$ apart from each other, at a distance of more than $250 \mathrm{~cm}$ from the observer. In setting $\Delta \beta$, the respective points were located about $80 \mathrm{~cm}$ apart from each other, and slightly closer to the observer. The differences $\Delta \alpha$ and $\Delta \beta$, however, do not only depend on these particular points, but also on the remaining points in each of the configurations. Figure 3 indicates a slight deviation of the data points from a line of constant monocular azimuth $\alpha$ and $\beta$, respectively. This deviation, however, is small compared with the variance that the data points exhibit along this line.

The results of the experiment and the performed statistical tests are summarized in Table 1 . The listed means differ from zero, the value predicted by the Reidemeister condition, in the magnitude of 0.012 to $5.76 \mathrm{arcmin}$. In most cases, the standard deviations are in a range of a few minutes of arc, too. Two different procedures were employed to evaluate the statistical significance of the means of $\Delta \alpha$ and $\Delta \beta$. The $p$ values that result from a standard parametric one-sample $t$ test against zero with the given degrees of freedom $d f$ are denoted by the symbol 


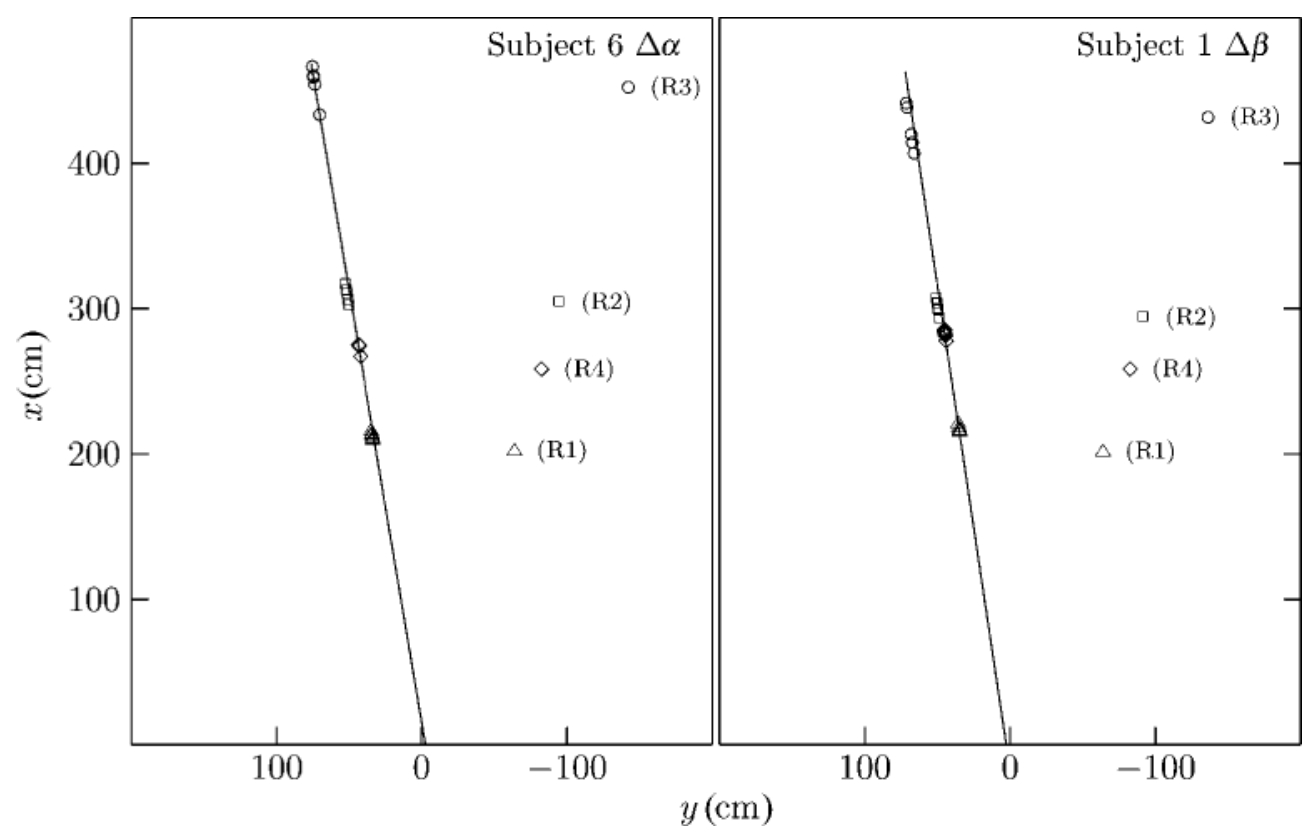

Figure 3. Graphical illustration of the results of Subject 6 (setting $\Delta \alpha$ ) and Subject 1 (setting $\Delta \beta$ ). The reference lines indicate the locus of constant coordinates $\alpha$ and $\beta$, respectively.

$p^{*}$. In the present context, however, the underlying assumption of a normal sampling distribution may be violated. Although the Reidemeister condition forces the differences $\Delta \alpha$ and $\Delta \beta$ to vanish for all situations considered in the experiment, changing configurations over sessions, which was necessary in some cases, will most likely affect the sampling distributions of these differences. Thus a randomization test (cf. Edgington, 1995; Good, 2000) was used, which does not rely on parametric assumptions. The values $p^{\dagger}$ provide the (exact) probabilities of obtaining a more extreme absolute $t$ value (or mean, respectively) than the one obtained under systematic permutations of the order of the two estimates from

Table 1

Results of All Subjects in Both Settings $(\Delta \alpha$ and $\Delta \beta)$

\begin{tabular}{cccccccc}
\hline Subject & Setting & $M$ & $S D$ & \multicolumn{1}{c}{$t$} & $d f$ & $p^{*}$ & $p^{\dagger}$ \\
\hline 1 & $\Delta \alpha$ & -0.028 & 0.046 & -1.332 & 4 & .254 & .313 \\
& $\Delta \beta$ & -0.026 & 0.027 & -2.181 & 4 & .095 & .125 \\
2 & $\Delta \alpha$ & -0.008 & 0.057 & -0.314 & 4 & .769 & .813 \\
& $\Delta \beta$ & -0.012 & 0.061 & -0.452 & 4 & .675 & .875 \\
3 & $\Delta \alpha$ & -0.036 & 0.029 & -2.545 & 3 & .084 & .000 \\
& $\Delta \beta$ & -0.008 & 0.046 & -0.376 & 4 & .726 & .563 \\
4 & $\Delta \alpha$ & -0.040 & 0.038 & -2.399 & 4 & .074 & .000 \\
& $\Delta \beta$ & 0.008 & 0.028 & 0.616 & 4 & .571 & .563 \\
5 & $\Delta \alpha$ & 0.001 & 0.081 & 0.037 & 4 & .972 & .875 \\
& $\Delta \beta$ & -0.064 & 0.055 & -2.616 & 4 & .059 & .063 \\
6 & $\Delta \alpha$ & -0.001 & 0.050 & -0.045 & 4 & .966 & .813 \\
& $\Delta \beta$ & -0.005 & 0.070 & -0.153 & 4 & .886 & .813 \\
7 & $\Delta \alpha$ & 0.020 & 0.161 & 0.249 & 3 & .819 & .875 \\
& $\Delta \beta$ & -0.004 & 0.058 & -0.154 & 4 & .885 & .813 \\
\hline
\end{tabular}

Note-Means and standard deviations are in degrees. $* p$ values resulting from a parametric $t$ test. ${ }^{\dagger} p$ values resulting from a nonparametric permutation test on the $t$ values. which the session-specific differences $\Delta \alpha$ and $\Delta \beta$ are computed. Statistical decisions will be based on the exact probability $p^{\dagger}$. A liberal significance level of 0.2 is chosen in order to control for Type II error. Under these conditions, the statistical decisions based on $p^{*}$, however, do not differ from those based on $p^{\dagger}$, except for two cases in which the $t$ test turns out to be more conservative.

Two significant values occur in each of the settings $\Delta \alpha$ and $\Delta \beta$, but for none of the subjects do the data significantly deviate from the predictions in both settings. For Subjects 2, 6, and 7, the data unequivocally speak to the validity of the Reidemeister condition because their results are nonsignificant in both settings. Notice that some of the resulting $p$ values are remarkably large (see Subject 2, for example).

\section{DISCUSSION}

The characteristic shape of the LPED in binocular vision is commonly taken as providing evidence for the influence of vergence-related binocular information (Foley, 1978, 1980). In contrast to this approach, Heller (1997) suggested a theoretical account based on the idea of combining the monocular information without any binocular interaction. It provides a straightforward generalization of the classic Luneburg theory (Luneburg, 1947) and is characterized by a set of qualitative and empirically testable axioms within a measurement-theoretic approach. This allows for testing the theory in its most general form and, in particular, avoids the necessity of specifying a psychophysical relationship. The present article is devoted to an experimental test of the key axiom, the 
Reidemeister condition, the rationale of which is illustrated in Figure 2. It amounts to checking whether two points, resulting from certain successive equidistant matches, have the same monocular direction.

The experimental results show that the discrepancies from the predictions of the Reidemeister condition, as well as the observed standard deviations, tend to be very small in most cases. In view of the relatively large stimulus configurations and the considerable distance between the points that enter into the statistical tests, this would not be expected if vergence were significantly affecting judgments of equidistance. At a liberal significance level of $\alpha=0.2$, which was chosen to limit the probability of Type II error, statistically significant deviations only occur in 4 out of 14 cases. Notice that the expected number of significant cases is 2.8 even if the null hypothesis holds. Thus, I interpret the results of the present study as an empirical validation of the Reidemeister condition.

From this, it may be concluded that neither relative nor absolute vergence is an effective depth cue in this experimental situation. Foley and Richards (1972) already have shown that results with static fixation do not differ from those obtained with eye movements allowed unless the parallax differences between the stimuli are larger than $1^{\circ}$. Thus, it is unlikely that fixating stimuli in turn provided effective oculomotor cues to relative depth, since points of equal egocentric distance will fall within this range in most cases. The ineffectiveness of absolute vergence is an immediate consequence of the Reidemeister condition, which constitutes a direct test of the assumption that the perceptually relevant monocular information does not depend on the absolute position of the stimuli. The Reidemeister condition implies that for any pair of stimuli $(\alpha, \beta),\left(\alpha^{\prime}, \beta^{\prime}\right)$, the perceptually relevant information in the left eye remains constant with the coordinates $\beta$ and $\beta^{\prime}$ fixed, independent of the respective $\alpha$ coordinates. This means that, even though absolute vergence varies with different $\alpha$ coordinates, there is no indication of a reevaluation of the monocular information related to the $\beta$ coordinates as suggested by Foley's $(1978,1980)$ theory. This result is in line with the widely accepted view that vergence is not a reliable depth cue at the distances of the presented stimuli, whereas Foley's account of the LPED heavily draws upon a perception of vergence that is not necessarily veridical but very sensitive. In general, the role of vergence may be different when stimuli are within arm's reach (see Mon-Williams \& Tresilian, 1999), and the validity of the Reidemeister condition remains to be tested under these circumstances. Due to the systematic discrepancy between the LPED and the Vieth-Müller circle, disparity and vergence provide slightly divergent information. Vergence may thus affect the equidistance judgments, although it is not necessary to evaluate absolute distance for solving the experimental task. However, I expect that, even in near space, the disparity cues will prevail over a more reliable yet imprecise vergence cue. This point of view is corroborated by Viguier, Clément, and Trotter (2001). They found that equidistance settings in the vicinity of a reference point located $60-80 \mathrm{~cm}$ in front of the observer were veridical only if disparity cues were available.

Another remarkable result is evident from Figure 3. Although the loci of perceived equidistance vary over a considerable range in radial direction, the variation of considered coordinate differences $\Delta \alpha$ and $\Delta \beta$ remains at a low level (see Table 1). Obviously, the performed experimental tests of the Reidemeister condition are not affected by the observed variation of the LPED. This clearly is a benefit of the employed measurement-theoretic approach, which is capable of eliminating from the analysis the variance due to monocular effects. The indifferences $\mathrm{R} 1$ to R4 characterize the assumed independence of the eyes in terms of qualitative empirical observations that are not tied to a particular choice of the monocular input transformations. Thus, the observed pattern of results is consistent with Equation 3 not only if the functions $f$ and $g$ are time invariant, but even if they vary over sessions.

The effects captured by the monocular input transformations may be caused by optical properties of the eye or by the neural processing that occurs prior to binocular combination. It is highly plausible that some of the systematic deviations to the Vieth-Müller circle are due to the oversimplified eye model that forms the basis of its derivation as a theoretical LPED. Asymmetries of the LPED with respect to the median plane, which may indicate aniseikonia (cf. Howard \& Rogers, 1995), can easily be explained by appropriately choosing the functions $f$ and $g$ in Equation 3. It can be shown that the LPED is symmetric if and only if $f(\alpha)=-g(-\alpha)+r$ holds with an arbitrary constant $r$. Other relevant optical factors may be the dissociation of optical node and rotation center of the eye (cf. Gulick \& Lawson, 1976), and the deviations of the retinas from a spherical form. Modeling these effects will contribute to determining the form of the functions $f$ and $g$ within a bottom-up approach. A top-down strategy attempts to limit the possible forms of these functions by applying functional equation techniques. Aczél, Boros, Heller, and Ng (1999) were able to prove that - under appropriate side conditions - only two distinct forms of the functions $f$ and $g$ are possible whenever the LPED is characterized by a constant pattern of differences to the Vieth-Müller circle in terms of binocular parallax. Both functions are either linear or of a special exponential form. These possibilities also cover the Luneburg theory, which holds if and only if the functions $f$ and $g$ both are linear with identical slopes. However, if the observed pattern of parallax differences is not constant, as the available data suggest, both forms can be excluded from consideration.

Figure 4 illustrates how closely the empirically observed LPED can be predicted by a specific choice of the functions $f$ and $g$ in Equation 3. The points in the graphs on the left show average equidistance settings to four reference points presented straight ahead for two subjects of Foley (1966). The solid curves represent the predictions of these data that are derived from the functions $f$ and $g$ depicted 

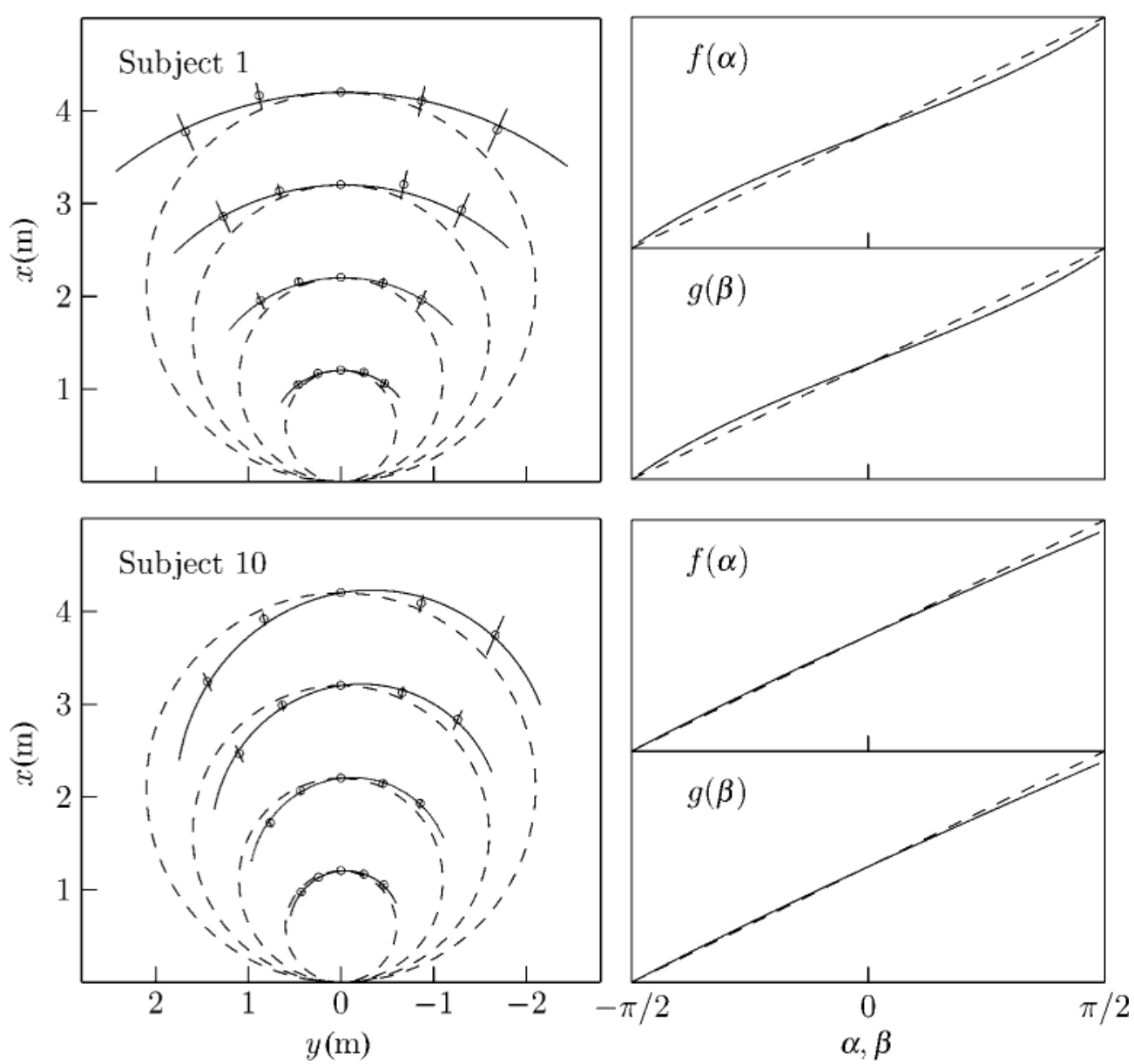

Figure 4. The graphs on the left show average equidistance settings to four reference points presented straight ahead for Subjects 1 and 10 of Foley (1966). Radial line segments indicate standard deviations. The solid curves represent the predictions derived from the functions $f$ and $g$, as illustrated by the graphs on the right.

in the graphs on the right (see Heller, 1997, for details on the functions and the applied goodness-of-fit criterion). Notice that a labeling of the ordinate can be omitted, because the functions are unique up to independent shifts of their origins and the choice of a common unit. Their shape may be interpreted with respect to the dashed reference line representing the identity function. A comparison of the upper and lower pair of graphs reveals that the curvilinear form of the functions $f$ and $g$ for Subject 1 is responsible for the LPED being less curved than the Vieth-Müller circle. In both cases, the plotted standard deviations indicate that the predictions are within experimental error.

It can be concluded that the theory proposed by Heller (1997) is not only capable of predicting the characteristic features of the empirically observed LPED (see Figure 4) but also passes the explicit test of its axiomatic foundation. Moreover, it allows for an integrated treatment of perceived egocentric distance and direction, which constitutes an important advantage over previous approaches. Usually, binocular direction is treated separately from egocentric radial distance (e.g., Blank, 1978; Foley, 1965, 1991). Defining

$$
\Gamma=f(\alpha)-g(\beta) \text { and } \Phi=\frac{f(\alpha)+g(\beta)}{2},
$$

however, yields a psychologically significant recoordinatization of physical space generalizing Equation 1. From the reported results, it follows that the coordinate $\Gamma$ characterizes the LPED. The trajectories of constant coordinates $\Gamma$ and $\Phi$ are related to each other in exactly the same way as are the Vieth-Müller circles and hyperbolas of Hillebrand (see Figure 1B), reflecting the relationship between the notions of corresponding and symmetrical retinal points. This motivates the consideration of $\Phi$ as a characterization of the locus of constant binocular direction, a hypothesis that has not yet been confirmed experimentally (mainly due to the difficulties of maintaining single vision with points of equal binocular direction). Notice that substituting $\Gamma, \Phi$ for $\gamma, \varphi$ at each 
of their occurrences in the Luneburg theory (Luneburg, 1947) leads to a straightforward generalization of that model.

The experimental results presented here challenge the view that the LPED depends on vergence-related oculomotor cues as proposed by Foley $(1978,1980)$. They demonstrate that Foley's account is by no means exclusively dictated by the data. From the perspective advocated in the present article, the supposed dependence of the LPED on binocular information is an artifact that arises from oversimplifying assumptions concerning the optics of the eye and the neural processing of monocular information. The theory of Heller (1997) provides a formal framework that is able to capture realistic assumptions with respect to these factors by monocular input transformations, the particular form of which remains to be determined.

\section{REFERENCES}

Aczél, J., Boros, Z., Heller, J., \& NG, C. T. (1999). Functional equations in binocular space perception. Journal of Mathematical Psychology, 43, 71-101.

BLANK, A. A. (1978). Metric geometry in human binocular perception: Theory and fact. In E. L. J. Leeuwenberg \& H. F. J. M. Buffart (Eds.), Formal theories of visual perception (pp. 83-102). New York: Wiley.

Edgington, E. S. (1995). Randomization tests (3rd ed.). New York: Dekker.

FoLEY, J. M. (1965). Visual space: A scale of perceived relative direction. Proceedings of the 73rd Annual Convention of the American Psychological Association, 49-50.

FolEY, J. M. (1966). Locus of perceived equidistance as a function of viewing distance. Journal of the Optical Society of America, 56, 822827.

FOLEY, J. M. (1970). Loci of perceived, equi-, half- and double-distance in stereoscopic vision. Vision Research, 10, 1201-1209.
Foley, J. M. (1978). Primary distance perception. In R. Held, H. W. Leibowitz, \& H.-L. Teuber (Eds.), Handbook of sensory physiology: Vol. VIII. Perception (pp. 181-213). Berlin: Springer-Verlag.

FoleY, J. M. (1980). Binocular distance perception. Psychological Review, 87, 411-434.

FoLEY, J. M. (1991). Binocular space perception. In D. Regan \& J. R. Cronly-Dillon (Eds.), Binocular vision: Vision and visual dysfunction, Vol. 9 (pp. 75-92). Basingstoke, U.K.: Macmillan.

Foley, J. M., \& Richards, W. (1972). Effects of voluntary eye movement and convergence on the binocular appreciation of depth. Perception \& Psychophysics, 11, 423-427.

Good, P. I. (2000). Permutation tests (2nd ed.). New York: SpringerVerlag.

Gulick, W. L., \& Lawson, R. B. (1976). Human stereopsis: A psychophysical analysis. New York: Oxford University Press.

Hardy, L. H., Rand, G., Rittler, M. C., Blank, A. A., \& Boeder, P. (1953). The geometry of binocular space perception. New York: Knapp Memorial Laboratories.

HeLler, J. (1997). On the psychophysics of binocular space perception. Journal of Mathematical Psychology, 41, 29-43.

HowARD, I. P., \& Rogers, B. J. (1995). Binocular vision and stereopsis. New York: Oxford University Press.

InDOw, T. (1991). A critical review of Luneburg's model with regard to global structure of visual space. Psychological Review, 98, 430-453.

Kesten, H. (1958). Accelerated stochastic approximation. Annals of Mathematical Statistics, 29, 41-59.

Krantz, D. H., Luce, R. D., Suppes, P., \& Tversky, A. (1971). Foundations of measurement (Vol. 1). New York: Academic Press.

Luneburg, R. K. (1947). Mathematical analysis of binocular vision. Princeton, NJ: Princeton University Press.

Mon-Williams, M., \& Tresilian, J. R. (1999). Some recent studies on the extraretinal contribution to distance perception. Perception, $\mathbf{2 8}$, 167-181.

RoBERTS, F. S., \& SUPPES, P. (1967). Some problems in the geometry of visual perception. Synthèse, 17, 171-201.

Viguier, A., Clément, G., \& Trotter, Y. (2001). Distance perception within near visual space. Perception, 30, 115-124.

(Manuscript received September 11, 2002; revision accepted for publication January 11, 2004.) 